\title{
NOTE ON THE REGION OF OVERCONVERGENCE OF DIRICHLET SERIES WITH OSTROWSKI GAPS
}

\author{
by J. P. EARL and J. R. SHACKELL
}

(Received 4 March, 1965)

1. The main object of this note is to show that a proof given by A. J. Macintyre [2] of a result on the overconvergence of partial sums of power series works more easily in the context of Dirichlet series. Applying this observation to the particular Dirichlet series $\sum a_{n} e^{-n s}$, we can remove certain restrictions which Macintyre finds necessary in the direct treatment of power series.

We consider a Dirichlet series

$$
\sum_{n=1}^{\infty} a_{n} e^{-\lambda_{n} s} \quad(s=\sigma+i t)
$$

where $\lambda_{n}=\mu_{n}+i v_{n}\left(\mu_{n}\right.$ and $v_{n}$ real), with $\mu_{n}$ increasing and tending to infinity and $v_{n}=o\left(\mu_{n}\right)$. We assume that the series has a finite abscissa of absolute convergence, which we may take to be $\sigma=0$. Our main result is then

THEOREM 1. Suppose (i) that $f(s)=\sum a_{n} e^{-\lambda_{n} s}$ has abscissa of absolute convergence $\sigma=0$, and is continuable in some neighbourhood of the origin throughout the angle $\phi_{1}<\arg s<\phi_{2}$; i.e. in the region $0<|s|<\delta, \phi_{1}<\arg s<\phi_{2}$ for some $\delta>0$, with $-\frac{3}{2} \pi<\phi_{1} \leqq-\frac{1}{2} \pi$, $\frac{1}{2} \pi \leqq \phi_{2}<\frac{3}{2} \pi$; (ii) $\lambda_{n}=\mu_{n}+i v_{n}$, where $\mu_{n}$ increases and tends to infinity and $v_{n}=o\left(\mu_{n}\right)$; (iii) there exists an increasing sequence of integers $\left\{n_{k}\right\}$, where $n_{k} \rightarrow \infty$ as $k \rightarrow \infty$, such that

$$
\frac{\mu_{n_{k}+1}}{\mu_{n_{k}}} \geqq 1+h,
$$

where $h>0$. Then, if $\phi_{1}^{\prime}, \phi_{2}^{\prime}$ are angles satisfying

$$
\phi_{1}<\phi_{1}^{\prime}<\phi_{2}^{\prime}<\phi_{2}
$$

there exists a neighbourhood of the origin in which

$$
\sum_{p=1}^{n_{k}} a_{p} e^{-\lambda_{p} s} \rightarrow f(s), \text { as } k \rightarrow \infty
$$

throughout the angle $\phi_{1}^{\prime} \leqq \arg s \leqq \phi_{r}^{\prime}$.

If $1 / \lambda_{n}=o(1 / \log n)$, then the abscissa of absolute convergence coincides with that of convergence.

Cases of particular interest occur when there is an easily approachable, or a virtually isolated, singularity at the origin. 
2. Proof of Theorem 1. Write

and

$$
S_{n}(s)=\sum_{p=1}^{n} a_{p} e^{-\lambda_{p} s}
$$

$$
R_{n}(s)=f(s)-S_{n}(s)
$$

We now obtain some estimates for $\left|R_{n}(s)\right|$.

LEMMA 1. If $\bar{D}$ is any compact subset of the domain of continuability of $f(s)=\sum a_{p} e^{-\lambda_{p} s}$ and $\gamma>0$, then for every $\varepsilon>0$ there exists $n_{0}(\varepsilon, \bar{D})$, such that, if $n \geqq n_{0}$,

(i) $\left\{\log \left|R_{n}(s)\right|\right\} / \mu_{n} \leqq-\sigma+\varepsilon$, for $\sigma \leqq 0, s$ in $\bar{D}$,

(ii) $\left\{\log \left|R_{n}(s)\right|\right\} / \mu_{n} \leqq \varepsilon, \quad$ for $\sigma>0, s$ in $\bar{D}$,

(iii) $\left\{\log \left|R_{n}(s)\right|\right\} / \mu_{n+1} \leqq-\sigma+\varepsilon$, for $\sigma \geqq \gamma>0$.

Proof. Case (i): $\sigma \leqq 0$. Suppose that $|t| \leqq T$ in $\bar{D}$ and define

Then $\omega_{n}=o\left(\mu_{n}\right)$,

$$
\omega_{n}=\sup _{1 \leqq p \leqq n}\left|v_{p}\right|
$$

$$
\left|S_{n}(s)\right| \leqq e^{\omega_{n}|t|-\mu_{n} \sigma} \sum_{p=1}^{n}\left|a_{p}\right|
$$

and since $\sum a_{p} e^{-\lambda_{p} s}$ is absolutely convergent for every $\sigma>0$ we have, with $\varepsilon_{1}=\frac{1}{4} \varepsilon$,

$$
e^{-\mu_{n} \varepsilon_{1}} \sum_{p=1}^{n}\left|a_{p}\right| \leqq \sum_{p=1}^{n}\left|a_{p}\right| e^{-\mu_{p} e_{1}} \leqq K(\varepsilon) .
$$

Therefore, for $n \geqq n_{1}(\varepsilon)$,

and

$$
\frac{1}{\mu_{n}} \log \left(\sum_{p=1}^{n}\left|a_{p}\right|\right) \leqq 2 \varepsilon_{1},
$$

for $n \geqq n_{2}(\varepsilon)$. Now write

$$
\begin{aligned}
\frac{1}{\mu_{n}} \log \left|S_{n}(s)\right| & \leqq-\sigma+2 \varepsilon_{1}+\frac{\omega_{n} T}{\mu_{n}} \\
& \leqq-\sigma+3 \varepsilon_{1},
\end{aligned}
$$

$$
M=\sup _{\bar{D}}\{|f(s)|\}
$$

Then

$$
\left\{\log \left|R_{n}(s)\right|\right\} / \mu_{n} \leqq\left\{\log \left(M+\left|S_{n}(s)\right|\right)\right\} / \mu_{n} \leqq-\sigma+4 \varepsilon_{1}=-\sigma+\varepsilon,
$$

for $n \geqq n_{3}(\varepsilon, \bar{D})$. 
Case (ii): $0<\sigma<\gamma$. Let $\varepsilon_{1}=\frac{1}{3} \varepsilon$. We have

Therefore

$$
\begin{aligned}
e^{-\mu_{n} e_{1}}\left|\sum_{p=1}^{n} a_{p} e^{-\lambda_{p} s}\right| & \leqq \sum_{p=1}^{n}\left|a_{p} e^{-\mu_{p}\left(s+\varepsilon_{1}\right)-i v_{p} s}\right| \\
& \leqq e^{\omega_{n} T} \sum_{p=1}^{n}\left|a_{p}\right| e^{-\mu_{p} \varepsilon_{1}} \\
& \leqq e^{\omega_{n} T} K(\varepsilon) .
\end{aligned}
$$

and, for $n \geqq n_{4}(\varepsilon, \bar{D})$,

$$
\left|\sum_{p=1}^{n} a_{p} e^{-\lambda_{p} s}\right| \leqq K(\varepsilon) \exp \left\{\omega_{n} T+\mu_{n} \varepsilon_{1}\right\}
$$

$$
\left\{\log \left|R_{n}(s)\right|\right\} / \mu_{n} \leqq 3 \varepsilon_{1}=\varepsilon:
$$

Case (iii): $\sigma \geqq \gamma>0$. Choose $\varepsilon<\frac{1}{2} \gamma, \varepsilon_{2}=\frac{1}{2} \varepsilon$. Since

$$
\begin{aligned}
R_{n}(s) & =\sum_{p=n+1}^{\infty} a_{p} e^{-\lambda_{p} s}, \\
\left|R_{n}(s)\right| & \leqq e^{-\mu_{n+1}} \sigma \sum_{p=n+1}^{\infty}\left|a_{p} e^{-i v_{p} s} e^{\left(\mu_{n+1}-\mu_{p}\right) s}\right| \\
& \leqq e^{-\mu_{n+1} \sigma} \sum_{p=n+1}^{\infty}\left|a_{p} e^{i v_{p} s}\right| e^{\left(\mu_{n+1}-\mu_{p}\right) \varepsilon_{2}} \\
& \leqq e^{-\mu_{n+1}\left(\sigma-\varepsilon_{2}\right)} \sum_{p=n+1}^{\infty}\left|a_{p}\right| e^{v_{p} t-\mu_{p} \varepsilon_{2}} \\
& \leqq K(\varepsilon) e^{-\mu_{n+1}\left(\sigma-\varepsilon_{2}\right)} .
\end{aligned}
$$

Therefore, for $n \geqq n_{5}(\varepsilon)$,

$$
\left\{\log \left|R_{n}(s)\right|\right\} / \mu_{n+1} \leqq-\sigma+2 \varepsilon_{2}=-\sigma+\varepsilon .
$$

This completes the proof of the lemma.

We show that, if $\phi_{2}^{\prime}<\phi_{2}$, then $S_{n_{k}}(s) \rightarrow f(s)$ in some neighbourhood of the origin, throughout the angle $-\frac{1}{2} \pi<\arg s \leqq \phi_{2}^{\prime}$. A similar argument shows that, if $\phi_{1}^{\prime}>\phi_{1}, S_{n_{k}}(s) \rightarrow f(s)$ in some neighbourhood of the origin throughout the angle $\phi_{1}^{\prime} \leqq \arg s<\frac{1}{2} \pi$. If $\phi_{2} \leqq \frac{1}{2} \pi$ we have nothing to prove, and hence we may assume that $\phi_{2}>\frac{1}{2} \pi$.

There exists a sequence $\left\{n_{k}\right\}$ such that $\mu_{n_{k}+1} / \mu_{n_{k}} \geqq 1+h$, where $h$ is a positive constant. From Lemma 1 we have, for every $\gamma>0$,

(i) $\left\{\log \left|R_{n_{k}}(s)\right|\right\} / \mu_{n_{k}} \leqq-\sigma+\varepsilon_{n_{k}}, \quad$ for $\sigma \leqq 0, s$ in $\bar{D}$,

(ii) $\left\{\log \left|R_{n_{k}}(s)\right|\right\} \mid \mu_{n_{k}} \leqq \quad \varepsilon_{n_{k}}, \quad$ for $\sigma>0, s$ in $\bar{D}$,

(iii) $\left\{\log \left|R_{n_{k}}(s)\right|\right\} / \mu_{n_{k}} \leqq-(1+h) \sigma+\varepsilon_{n_{k}}$, for $\sigma \geqq \gamma>0$,

where $\varepsilon_{n_{k}} \rightarrow 0$ as $k \rightarrow \infty$. Now $f(s)$ is regular in some neighbourhood of the origin throughout an angle $-\frac{1}{2} \pi<\arg s<\phi_{2}$, where $\frac{1}{2} \pi<\phi_{2}<\frac{3}{2} \pi$. Then we may choose $\delta>0$ such that $f(s)$ 
is regular in $0<|s|<2 \delta,-\frac{1}{2} \pi<\arg s<\phi_{2}$. Hence, for every $\eta$ in $0<\eta<\delta, f(s)$ is regular in the region $\bar{D}_{\eta}$, where $D_{\eta}$ is the half disc

$$
|s-i \eta|<\delta, \quad \phi_{2}-\pi<\arg (s-i \eta)<\phi_{2} .
$$

Let $\Gamma_{\eta}$ be the boundary of $D_{\eta}$. Now $\left\{\log \left|R_{n_{k}}(s)\right|\right\} / \mu_{n_{k}}$ is a subharmonic function; hence if $u_{n_{k}}(s)$ is function harmonic in $D_{\eta}$ and taking on $\Gamma_{\eta}$ boundary values $-\sigma+\varepsilon_{n_{k}}$ for $\sigma \leqq 0, \varepsilon_{n_{k}}$ for $0<\sigma<\gamma$, and $-(1+h) \sigma+\varepsilon_{n_{k}}$ for $\sigma \geqq \gamma$, then $\left\{\log \left|R_{n_{k}}(s)\right|\right\} / \mu_{n_{k}} \leqq u_{n_{k}}(s)$ in $D_{\eta}$. As $k \rightarrow \infty, \varepsilon_{n_{k}} \rightarrow 0$ and $u_{n_{k}}(s) \rightarrow u(s)$, where $u(s)$ is harmonic in $D_{\eta}$ and takes on $\Gamma_{\eta}$ the boundary values $-\sigma$ for $\sigma \leqq 0,0$ for $0<\sigma<\gamma$, and $-(1+h) \sigma$ for $\sigma \geqq \gamma$. Also in $D_{\eta}$

$$
\limsup _{k \rightarrow \infty} \frac{1}{\mu_{n_{k}}} \log \left|R_{n_{k}}(s)\right| \leqq u(s) \text {. }
$$

If $u(s)<0$, then $R_{n_{k}}(s) \rightarrow 0$ and so $S_{n_{k}}(s) \rightarrow f(s)$.

We can take $\gamma$ as small as we please, and therefore $u(s)$ may be taken to differ by as little as we please from $v(s)$, where $v(s)$ is harmonic in $D_{\eta}$ and takes on $\Gamma_{\eta}$ the boundary values $-\sigma$ for $\sigma \leqq 0$ and $-(1+h) \sigma$ for $\sigma>0$.

We now take a new variable $z=r e^{i \theta}=(s-i \eta) e^{i\left(\phi_{2}-\pi\right)}$. Consider $\operatorname{Im}\{z \log z\}$, which is harmonic for $\operatorname{Im} z>0$ and takes on $y=\operatorname{Im} z=0$ the boundary values $\pi x$ for $x=\operatorname{Re} z<0$ and 0 for $x \geqq 0$.

Let $v^{*}(z)=v(s)$. Consider

$$
g(z)=v^{*}(z)-(h / \pi) \cos \left(\phi_{2}-\pi\right) \operatorname{Im}\{z \log z\}+(1+h) x \cos \left(\phi_{2}-\pi\right),
$$

which is harmonic for $s$ in $D_{\eta}$ and zero on $y=0$. Hence for $s$ in $D_{\eta}$,

$$
g\left(r e^{i \theta}\right)=\frac{1}{2 \pi} \int_{0}^{\pi} g\left(\delta e^{i \theta}\right) \frac{4\left(\delta^{2}-r^{2}\right) \delta r \sin \theta \sin \phi}{\left[\delta^{2}-2 \delta r \cos (\theta-\phi)+r^{2}\right]\left[\delta^{2}-2 \delta r \cos (\theta+\phi)+r^{2}\right]} d \phi,
$$

and therefore

$$
\begin{aligned}
\left|g\left(r e^{i \theta}\right)\right| & \leqq r \sin \theta \cdot \frac{2(\delta+r)}{(\delta-r)^{3}} \sup _{0 \leqq \phi \leqq \pi}\left|g\left(\delta e^{i \phi}\right)\right| \\
& \leqq K\left(r_{0}\right) r \sin \theta
\end{aligned}
$$

for $r \leqq r_{0}<\delta$. Therefore

$$
\begin{aligned}
v^{*}\left(r e^{i \theta}\right)= & H(r, \theta) r \sin \theta+(h / \pi) \cos \left(\phi_{2}-\pi\right) \operatorname{Im}\left\{r e^{i \theta} \log \left(r e^{i \theta}\right)\right\}-(1+h) r \cos \theta \cos \left(\phi_{2}-\pi\right), \\
= & r\left\{H(r, \theta) \sin \theta+(h / \pi) \cos \left(\phi_{2}-\pi\right) \sin \theta \log r+(\theta h / \pi) \cos \theta \cos \left(\phi_{2}-\pi\right)\right. \\
& \left.-(1+h) \cos \theta \cos \left(\phi_{2}-\pi\right)\right\},
\end{aligned}
$$

where $H(r, \theta)$ is bounded.

Now $\log r \rightarrow-\infty$ as $r \rightarrow 0+$. Hence for every $\theta$ in $0<\theta<\pi$ there exists $r_{0}(\theta)$ such that, if $r \leqq r_{0}(\theta), v^{*}\left(r e^{i \theta}\right)<0$. Thus $v$ is negative in a region $R_{\eta}$, fixed with respect to $D_{\eta}$, the boundary of which touches $\Gamma_{\eta}$ at $z=0$, and $R_{n_{k}}(s) \rightarrow 0$ as $k \rightarrow \infty$ at any point of $R_{\eta}$.

Let $\eta \rightarrow 0$; then $R_{\eta} \rightarrow R$, where $R$ is a region whose boundary touches the line arg $s=\phi_{2}$ at $s=0$. This is sufficient to establish Theorem 1 . 
3. As an almost immediate consequence of Theorem 1 we have

THEOREM 2. (Bourion [1]) If $\sum a_{n} z^{n}=f(z)$ is a power series with radius of convergence unity such that

$$
a_{n}=0 \text { for } n_{k}<n \leqq N_{k},
$$

where $\left\{n_{k}\right\}$ and $\left\{N_{k}\right\}$ are two sequences of integers such that

$$
\frac{N_{k}}{n_{k}} \geqq 1+h>1
$$

and if $f(z)$ is regular near $z=1$ for $-\alpha_{1}<\arg (1-z)<\alpha_{2}$, then the sequence $\left\{S_{n_{k}}(z)\right\}$ of partial sums converges to $f(z)$ in some neighbourhood of $z=1$ in the angle $-\beta_{1} \leqq \arg (1-z) \leqq \beta_{2}$, provided that $-\alpha_{1}<-\beta_{1}<\frac{1}{2} \pi$ and $\frac{1}{2} \pi<\beta_{2}<\alpha_{2}$.

As mentioned in the introduction, this follows from consideration of the Dirichlet series $\sum a_{n} e^{-n s}$ and the conformal map $z=e^{-s}$.

Macintyre's work concerned only the case $\alpha_{1}>\pi, \alpha_{2}>\pi$, and he showed that there exist angles $\gamma_{1}$ and $\gamma_{2}$, each depending on the value of $h$, satisfying

$$
\alpha_{1}>\gamma_{1}>\pi, \quad \alpha_{2}>\gamma_{2}>\pi,
$$

such that $S_{n_{\mathrm{k}}}(z) \rightarrow f(z)$ in some neighbourhood of $z=1$ throughout the angle

$$
-\gamma_{1}<\arg (1-z)<\gamma_{2} \text {. }
$$

4. We note that Theorems A and B of [2] can be established under slightly weaker conditions by using Bourion's result. Instead of requiring $f(z)$ to be continuable across the real axis $z>1$, from the upper half-plane into a definite angle $0>\arg (z-1)>-\left(\alpha_{1}-\pi\right)$ of the lower half-plane, and similarly from the lower half-plane into an equal angle of the upper half-plane, all that is needed is that the regions of continuability overlap in a neighbourhood of $z=1$ throughout some definite angle outside $|z| \leqq 1$.

Finally we wish to express our gratitude to M. E. Noble for his help and guidance, to the referee and editor of these Proceedings for their help in the presentation of this note, and to D.S.I.R. for financial support during the period involved.

\section{REFERENCES}

1. G. Bourion, L'ultraconvergence dans les séries de Taylor, Actualités Scientifiques et Industrielles no. 472 (Paris 1937).

2. A. J. Macintyre, An overconvergence theorem of G. Bourion and its application to coefficients of certain power series, Ann. Acad. Sci. Fenn. Ser. A.I. 250/23 (1958).

UNiversity OF Kent at CANTERbury 